\title{
Renormalization group of the Domany-Kinzel cellular automaton
}

\author{
Tânia Tomé and Mário J. de Oliveira \\ Instituto de Física \\ Universidade de São Paulo \\ Caixa Postal 66318 \\ 05315-970 São Paulo, São Paulo, Brazil
}

September 30, 2018

\begin{abstract}
We apply the dinamically driven renormalization group to study the critical properties of the Domany-Kinzel probabilistic cellular automaton. To preserve the absorbing state clusters with at least one site occupied are renormalized into a one occupied site and clusters with all sites empty are renormalized into a one empty site. We have obtained the phase diagram as well as the critical exponent related to the spatial correlation length.
\end{abstract}




\section{Introduction}

Among the variety of irreversible models that describe a nonequilibrium phase transition from an active state to an absorbing state, the DomanyKinzel probabilistic cellular automaton [1] together with the contact process [2] are perhaps the simplest ones. According to Janssen [3] and Grassberger [1], any one component model displaying a transition from an absorbing to an active state belongs in the same universality class as direct percolation. In fact, the Domany-Kinzel cellular automaton is equivalent to direct percolation.

The Domany-Kinzel probabilistic cellular automaton is defined as follows. In a one dimensional lattice each site can be in one of two states, either empty or occupied. At each time step the sites are updated simultaneously and independently. The state of a given site depends only on the states of two neighboring sites in the previous time step. The rules are: 1) if the two sites are both empty, the given site will be empty, 2) if one site is occupied and the other is empty, the given site will be occupied with probability $p_{1}$, and 3 ) if the two sites are both occupied, the given site will be occupied with probability $p_{2}$. Due to the rule number 1 , the state with all sites empty will be an absorbing state.

In the stationary state the Domany-Kinzel automaton exhibits two states: the absorbing state with all sites empty and an active state with a nonzero density of occupied sites. For small values of $p_{1}$ the system is in the absorbing state. When the parameter $p_{1}$ is increased the absorbing state becomes unstable, for sufficiently large values of $p_{1}$, given rise to an active state. The phase transition from the absorbing to the active state is a continuous transition and belongs to the same universality class as direct percolation.

Renormalization group ( $R G$ ) techniques in real space have been used successfully in studying the critical behavior of equilibrium statistical models since the work of Niemeyer and van Leeuwen [5]. In these techniques small clusters of sites are renormalized into just one site according to certain rules. For the case of the Ising model with the up-down symmetry, the most important rule is the majority rule which preserves the up-down symmetry of the model at each step of the renormalization. Any RG scheme should be set up in a way to preserve the symmetries and the main properties of 
the model studied. In the present case the most important property is the existence of an absorbing state which should then be preserved by the RG trasformation. To this end, we have constructed a real space RG such that a cluster with at least one site occupied is renormalized into a one occupied site. Only clusters with all sites empty are renormalized into a one empty site.

For systems in equilibrium, that is, for systems defined by a Hamiltonian the appropriate RG space is the space of the parameters, or coupling constants, that define the Hamiltonian. A sequence of RG transformations correspond to a trajectory in this space. Systems far from equilibrium, that is, systems that lack detailed balance, on the other hand, are not defined by Hamiltonians but by the dynamic rules or equivalently by the transition probabilities that govern its time evolution. In this case the appropriate RG space is the space of parameters that define the transition probabilities.

Here we use a real space RG [6] [7] [8] that renormalizes the transition probabilities so that one obtains a $R G$ transformation in the space spanned by the parameters that define the transition probabilities. In the case of the Domany-Kinzel cellular automaton studied here this space will be the space defined by the parameters $p_{1}$, and $p_{2}$. The scheme we use is an application of the dynamically driven renormalization group introduced by Pietronero et al. [9] and Vespignani et al. [10] [11] to study systems in critical states. A study of the Domany-Kinzel by means of a phenomenological RG has been done by Bagnoli et al. [12].

The RG transformation was accomplished by using cells of size $b=2$ and by using a series of approximations to the stationary state. As we increase the order of the approximation better values were obtained for the universal critical exponent as well as for the nonuniversal critical quantities. Our best value for the critical exponent $\nu_{\perp}$ related to spatial correlation length is $\nu_{\perp}=1.013$ which should be compared to $\nu_{\perp}=1.100 \pm 0.005$ obtained from transfer matrix methods [1]. The critical value of $p_{1}$ along the line $p_{2}=0$ is $p_{c}=0.792$ which is close to $p_{c}=0.799 \pm 0.002$ obtained from simulations |13.

\section{RG transformation}

Consider a one dimensional lattice of $N$ sites in which each site can be in one of two states, either empty $\left(\sigma_{i}=0\right)$ or occupied $\left(\sigma_{i}=1\right)$. At each 
time step the sites are updated simultaneously, and independently, so that the transition probability $W\left(\sigma \mid \sigma^{\prime}\right)$ from state $\sigma^{\prime}=\left(\sigma_{1}^{\prime}, \sigma_{2}^{\prime}, \ldots, \sigma_{N}^{\prime}\right)$ to state $\sigma=\left(\sigma_{1}, \sigma_{2}, \ldots, \sigma_{N}\right)$ is given by

$$
W\left(\sigma \mid \sigma^{\prime}\right)=\prod_{i} w\left(\sigma_{i} \mid \sigma_{i}^{\prime}, \sigma_{i+1}^{\prime}\right)
$$

where

$$
w(1 \mid 00)=0, \quad w(1 \mid 10)=w(1 \mid 01)=p_{1}, \quad w(1 \mid 11)=p_{2} .
$$

These rules completely defines the Domany-Kinzel probabilistic cellular automaton since

$$
w\left(0 \mid \sigma_{i}^{\prime}, \sigma_{i+1}^{\prime}\right)+w\left(1 \mid \sigma_{i}^{\prime}, \sigma_{i+1}^{\prime}\right)=1 .
$$

We remark that the expression (11) implies that, given the state $\sigma^{\prime}$, the variables $\sigma_{i}$ are independent random variables which in turn is equivalent to say that each site should be updated independently of the others, as desired. We call this property of the cellular automaton one-site independence.

In the stationary state the following condition

$$
P(\sigma)=\sum_{\sigma^{\prime}} W^{\ell}\left(\sigma \mid \sigma^{\prime}\right) P\left(\sigma^{\prime}\right)
$$

holds for any value of $\ell$, where $P(\sigma)$ is the stationary probability. If we use the notation $P_{\ell}\left(\sigma, \sigma^{\prime}\right)$ for the probability of having state $\sigma^{\prime}$ at a given time and state $\sigma$ at a $\ell$ time steps later then

$$
P_{\ell}\left(\sigma, \sigma^{\prime}\right)=W^{\ell}\left(\sigma \mid \sigma^{\prime}\right) P\left(\sigma^{\prime}\right)
$$

Similarly,

$$
\widetilde{P}\left(\tau, \tau^{\prime}\right)=\widetilde{W}\left(\tau \mid \tau^{\prime}\right) \widetilde{P}\left(\tau^{\prime}\right)
$$

is the analog equation for the renormalized system where $\widetilde{W}\left(\tau \mid \tau^{\prime}\right)$ is the (onestep) renormalized transition probability we are looking for and $\tau=\left(\tau_{1}, \tau_{2}\right.$, $\left.\ldots, \tau_{N^{\prime}}\right)$, with $N^{\prime}=N / b$, denotes a renormalized state.

Let $\mathcal{R}(\tau \mid \sigma)$ be a conditional probability of the renormalized state $\tau$ given the state $\sigma$, which has the following properties

$$
\mathcal{R}(\tau \mid \sigma) \geq 0, \quad \quad \sum_{\tau} \mathcal{R}(\tau \mid \sigma)=1
$$


The RG transformations are obtained by demanding that [6]

$$
\widetilde{P}\left(\tau, \tau^{\prime}\right)=\sum_{\sigma} \sum_{\sigma^{\prime}} \mathcal{R}(\tau \mid \sigma) \mathcal{R}\left(\tau^{\prime} \mid \sigma^{\prime}\right) P_{\ell}\left(\sigma, \sigma^{\prime}\right)
$$

from which follows

$$
\widetilde{P}\left(\tau^{\prime}\right)=\sum_{\sigma^{\prime}} \mathcal{R}\left(\tau^{\prime} \mid \sigma^{\prime}\right) P\left(\sigma^{\prime}\right) .
$$

This last expression is the one used in real space $R G$ for equilibrium systems. Taking the ratio of the last two equations we obtain [11]

$$
\widetilde{W}\left(\tau \mid \tau^{\prime}\right)=\frac{\sum_{\sigma} \sum_{\sigma^{\prime}} \mathcal{R}(\tau \mid \sigma) W^{\ell}\left(\sigma \mid \sigma^{\prime}\right) \mathcal{R}\left(\tau^{\prime} \mid \sigma^{\prime}\right) P\left(\sigma^{\prime}\right)}{\sum_{\sigma^{\prime}} \mathcal{R}\left(\tau^{\prime} \mid \sigma^{\prime}\right) P\left(\sigma^{\prime}\right)},
$$

which is the desired expression for the renormalized transition probability. Due to the presence of the stationary probability on the right hand side of (10), this is not a transformation that involves only the transition probability. However, if we use an approximation for the steady state $P(\sigma)$, such as a mean field approximation, then equation (10) provides a well defined RG transformation $W \rightarrow \widetilde{W}$ from the old $W$ to the new $\widetilde{W}$ transition probability.

It is interesting to write equation (10) in the form

$$
\widetilde{W}\left(\tau \mid \tau^{\prime}\right)=\sum_{\sigma} \sum_{\sigma^{\prime}} \mathcal{R}(\tau \mid \sigma) W^{\ell}\left(\sigma \mid \sigma^{\prime}\right) \mathcal{S}\left(\sigma^{\prime} \mid \tau^{\prime}\right)
$$

where

$$
\mathcal{S}\left(\sigma^{\prime} \mid \tau^{\prime}\right)=\frac{\mathcal{R}\left(\tau^{\prime} \mid \sigma^{\prime}\right) P\left(\sigma^{\prime}\right)}{\sum_{\sigma^{\prime}} \mathcal{R}\left(\tau^{\prime} \mid \sigma^{\prime}\right) P\left(\sigma^{\prime}\right)} .
$$

From this last expression it is clear that $\mathcal{S}\left(\sigma^{\prime} \mid \tau^{\prime}\right)$ is normalized with respect to the variables $\sigma^{\prime}$ and that it can be interpreted as a conditional probability of $\sigma^{\prime}$ given $\tau^{\prime}$. In this way it becomes quite obvious that the renormalized transition probability $\widetilde{W}\left(\tau \mid \tau^{\prime}\right)$ is properly normalized with respect to the variables $\tau$ as it should. One important property of $\mathcal{S}$ follows from a specific property of $\mathcal{R}$. We will see in the next section that $\mathcal{R}(\tau \mid \sigma)$ is either zero or one from which follows that $\mathcal{R}\left(\tau^{\prime} \mid \sigma\right) \mathcal{R}(\tau \mid \sigma)=\delta\left(\tau^{\prime}, \tau\right) \mathcal{R}(\tau \mid \sigma)$. Using this last equality we can check that

$$
\sum_{\sigma} \mathcal{R}(\tau \mid \sigma) \mathcal{S}\left(\sigma \mid \tau^{\prime}\right)=\delta\left(\tau^{\prime}, \tau\right),
$$

independently of $P(\sigma)$. 
We remark that we should not expect, in general, that, given the state $\tau^{\prime}$, the variables $\tau_{i}$ be independent. In other words, the stochastic process defined by $\widetilde{W}\left(\tau \mid \tau^{\prime}\right)$, given by equation (10), will not have the desired one-site independence. This property, however, can be accomplished in an approximate way as follows. Let us define the one-site marginal transition probability $\widetilde{w}\left(\tau_{i} \mid \tau^{\prime}\right)$ by

$$
\widetilde{w}\left(\tau_{i} \mid \tau^{\prime}\right)=\sum_{\tau}^{\prime} \widetilde{W}\left(\tau \mid \tau^{\prime}\right),
$$

where the sum is over all $\tau_{j}$ except $\tau_{i}$. Next we construct a transition probability $\widetilde{W}_{R}\left(\tau \mid \tau^{\prime}\right)$ as being the product of these one-site transition probabilities, that is, we define

$$
\widetilde{W}_{R}\left(\tau \mid \tau^{\prime}\right)=\prod_{i} \widetilde{w}\left(\tau_{i} \mid \tau^{\prime}\right)
$$

Of course $\widetilde{W}_{R}\left(\tau \mid \tau^{\prime}\right)$ does not coincide, in general, with $\widetilde{W}\left(\tau \mid \tau^{\prime}\right)$.

The final step in the renormalization procedure is to say that the renormalized cellular automaton will be defined by the transition probability $\widetilde{W}_{R}\left(\tau \mid \tau^{\prime}\right)$ which in turn has the form of equation (1). The last step is an approximation which we call the one-site independence approximation. In this manner we obtain a renormalized probabilistic cellular automaton, with the property of one-site independence, whose one-site transition probability $\widetilde{w}\left(\tau_{i} \mid \tau^{\prime}\right)$ is obtained from the original one $w\left(\sigma_{i} \mid \sigma^{\prime}\right)$ through equations (14), (10), and (1). Thus we understand the present renormalization as the transformation $w\left(\sigma_{i} \mid \sigma^{\prime}\right) \rightarrow \widetilde{w}\left(\tau_{i} \mid \tau^{\prime}\right)$.

\section{Renormalization algorithm}

We use a renormalization $\mathcal{R}(\tau \mid \sigma)$ in the form of a product which for the case $b=2$ reads

$$
\mathcal{R}(\tau \mid \sigma)=\prod_{k=1}^{N / 2} R\left(\tau_{k} \mid \sigma_{2 k}, \sigma_{2 k+1}\right),
$$

where

$$
R(1 \mid 00)=0, \quad R(1 \mid 10)=R(1 \mid 01)=R(1 \mid 11)=1
$$

and

$$
R(0 \mid 00)=1, \quad R(0 \mid 10)=R(0 \mid 01)=R(0 \mid 11)=0 .
$$

The rule $R(0 \mid 00)=1$ ensures that the state with all sites empty will be again an absorbing state after the renormalization transformation. 
To calculate the renormalized transition probability $\widetilde{w}\left(\tau_{1} \mid \tau_{2}, \tau_{3}\right)$ we consider a cluster of nine sites of the original space-time lattice: two sites (labeled 1 and 2 ) at a time $\ell$, three (labeled 3, 4 and 5) at time $\ell-1$, and four (labeled $6,7,8$ and 9$)$ at time $\ell-2$. The renormalization of the sites are as follows: $\left(\sigma_{1}, \sigma_{2}\right) \rightarrow \tau_{1},\left(\sigma_{6}, \sigma_{7}\right) \rightarrow \tau_{2}$, and $\left(\sigma_{8}, \sigma_{9}\right) \rightarrow \tau_{3}$. Using equations (14), (12), and (11), the renormalized transition probability $\widetilde{w}\left(\tau_{1} \mid \tau_{2}, \tau_{3}\right)$ are calculated by

$$
\begin{gathered}
\widetilde{w}\left(\tau_{1} \mid \tau_{2}, \tau_{3}\right)=\sum_{\sigma_{1}} \sum_{\sigma_{2}} \sum_{\sigma_{6}} \sum_{\sigma_{7}} \sum_{\sigma_{8}} \sum_{\sigma_{9}} R\left(\tau_{1} \mid \sigma_{1}, \sigma_{2}\right) w_{2}\left(\sigma_{1}, \sigma_{2} \mid \sigma_{6}, \sigma_{7}, \sigma_{8}, \sigma_{9}\right) \times \\
\times \rho\left(\sigma_{6}, \sigma_{7}, \sigma_{8}, \sigma_{9} \mid \tau_{2}, \tau_{3}\right),
\end{gathered}
$$

where

$$
\begin{gathered}
w_{2}\left(\sigma_{1}, \sigma_{2} \mid \sigma_{6}, \sigma_{7}, \sigma_{8}, \sigma_{9}\right)= \\
=\sum_{\sigma_{3}} \sum_{\sigma_{4}} \sum_{\sigma_{5}} w\left(\sigma_{1} \mid \sigma_{3}, \sigma_{4}\right) w\left(\sigma_{2} \mid \sigma_{4}, \sigma_{5}\right) w\left(\sigma_{3} \mid \sigma_{6}, \sigma_{7}\right) w\left(\sigma_{4} \mid \sigma_{7}, \sigma_{8}\right) w\left(\sigma_{5} \mid \sigma_{8}, \sigma_{9}\right),
\end{gathered}
$$

and

$$
\rho\left(\sigma_{6}, \sigma_{7}, \sigma_{8}, \sigma_{9} \mid \tau_{2}, \tau_{3}\right)=\frac{1}{\widetilde{P}\left(\tau_{2}, \tau_{3}\right)} R\left(\tau_{2} \mid \sigma_{6}, \sigma_{7}\right) R\left(\tau_{3} \mid \sigma_{8}, \sigma_{9}\right) P\left(\sigma_{6}, \sigma_{7}, \sigma_{8}, \sigma_{9}\right),
$$

with

$$
\widetilde{P}\left(\tau_{2}, \tau_{3}\right)=\sum_{\sigma_{6}} \sum_{\sigma_{7}} \sum_{\sigma_{8}} \sum_{\sigma_{9}} R\left(\tau_{2} \mid \sigma_{6}, \sigma_{7}\right) R\left(\tau_{3} \mid \sigma_{8}, \sigma_{9}\right) P\left(\sigma_{6}, \sigma_{7}, \sigma_{8}, \sigma_{9}\right) .
$$

To actually use the above equations we have to calculate in each step of the renormalization the stationary probability distribution $P(\sigma)$ related to the old transition probability $w\left(\sigma_{i} \mid \sigma_{i}^{\prime}, \sigma_{i+1}^{\prime}\right)$ which satisfies the balance equation

$$
P(\sigma)=\sum_{\sigma^{\prime}} W\left(\sigma \mid \sigma^{\prime}\right) P\left(\sigma^{\prime}\right),
$$

where $W\left(\sigma \mid \sigma^{\prime}\right)$ is given by (11). Of course, this equation cannot be solved exactly and we should seek for approximate solutions.

The simplest approximate solution of (23) is obtained by writing the following equation for the one-site probability

$$
P\left(\sigma_{1}\right)=\sum_{\sigma_{1}^{\prime}} \sum_{\sigma_{2}^{\prime}} w\left(\sigma_{1} \mid \sigma_{1}^{\prime}, \sigma_{2}^{\prime}\right) P\left(\sigma_{1}^{\prime}, \sigma_{2}^{\prime}\right),
$$


and by inserting, in the right hand side of it, the following approximation for the two-site probability

$$
P\left(\sigma_{1}, \sigma_{2}\right)=P\left(\sigma_{1}\right) P\left(\sigma_{2}\right) .
$$

The equation becomes then a closed equation which can then be solved by repeated iterations.

Better approximations can be set up by generalizing this procedure. An approximation of order $n$ is obtained as follows. From equation (23) we write down an equation for the $n$-site probability distribution, namely

$$
\begin{gathered}
P\left(\sigma_{1}, \sigma_{2}, \ldots, \sigma_{n}\right)= \\
=\sum_{\sigma_{1}^{\prime}} \ldots \sum_{\sigma_{n}^{\prime}} \sum_{\sigma_{n+1}^{\prime}} w\left(\sigma_{1} \mid \sigma_{1}^{\prime}, \sigma_{2}^{\prime}\right) w\left(\sigma_{2} \mid \sigma_{2}^{\prime}, \sigma_{3}^{\prime}\right) \ldots w\left(\sigma_{n} \mid \sigma_{n}^{\prime}, \sigma_{n+1}^{\prime}\right) P\left(\sigma_{1}^{\prime}, \sigma_{2}^{\prime}, \ldots \sigma_{n}^{\prime}, \sigma_{n+1}^{\prime}\right) .
\end{gathered}
$$

Next we use the following approximation [14] [15] for the $(n+1)$-site probability

$$
P\left(\sigma_{1}, \sigma_{2}, \sigma_{3}, \ldots, \sigma_{n}, \sigma_{n+1}\right)=\frac{P\left(\sigma_{1}, \sigma_{2}, \ldots, \sigma_{n}\right) P\left(\sigma_{2}, \sigma_{3}, \ldots, \sigma_{n+1}\right)}{P\left(\sigma_{2}, \sigma_{3}, \ldots, \sigma_{n}\right)},
$$

and insert it in the right hand side of (26). Then equation (26) becomes a closed equation for the $n$-site probability distribution since

$$
P\left(\sigma_{2}, \sigma_{3}, \ldots, \sigma_{n}\right)=\sum_{\sigma_{1}} P\left(\sigma_{1}, \sigma_{2}, \ldots, \sigma_{n}\right)
$$

If the order of approximation $n \geq 4$, then $P\left(\sigma_{1}, \sigma_{2}, \sigma_{3}, \sigma_{4}\right)$ is obtained from $P\left(\sigma_{2}, \sigma_{3}, \ldots, \sigma_{n}\right)$ by summing over all $\sigma_{i}$ except $\sigma_{1}, \sigma_{2}, \sigma_{3}$, and $\sigma_{4}$. When $n<4$, then for $n=1$,

$$
P\left(\sigma_{1}, \sigma_{2}, \sigma_{3}, \sigma_{4}\right)=P\left(\sigma_{1}\right) P\left(\sigma_{2}\right) P\left(\sigma_{3}\right) P\left(\sigma_{4}\right),
$$

for $n=2$,

$$
P\left(\sigma_{1}, \sigma_{2}, \sigma_{3}, \sigma_{4}\right)=\frac{P\left(\sigma_{1}, \sigma_{2}\right) P\left(\sigma_{2}, \sigma_{3}\right) P\left(\sigma_{3}, \sigma_{4}\right)}{P\left(\sigma_{2}\right) P\left(\sigma_{3}\right)},
$$

and for $n=3$,

$$
P\left(\sigma_{1}, \sigma_{2}, \sigma_{3}, \sigma_{4}\right)=\frac{P\left(\sigma_{1}, \sigma_{2}, \sigma_{3}\right) P\left(\sigma_{2}, \sigma_{3}, \sigma_{4}\right)}{P\left(\sigma_{2}, \sigma_{3}\right)}
$$




\section{Results}

The renormalization transformation defined in the previous sections may be sought of as a trajectory in the space spanned by the parameter $p_{1}$, and $p_{2}$. It can be easily checked that $\widetilde{w}(1 \mid 0,0)=0$ implying that a system with one absorbing state renormalizes into another one with the same property. Within the space of parameters $p_{1}$ and $p_{2}$, the renormalization transformation may be seen as a mapping $\left(p_{1}, p_{2}\right) \rightarrow\left(p_{1}^{\prime}, p_{2}^{\prime}\right)$. Given $w(1 \mid 10)=w(1 \mid 01)=p_{1}$, $w(1 \mid 11)=p_{2}$, and $w(1 \mid 0,0)=0$ we obtain $p_{1}^{\prime}=\widetilde{w}(1 \mid 10)$ and $p_{2}^{\prime}=\widetilde{w}(1 \mid 11)$ by using equation (19). It can be easily checked that $\widetilde{w}(1 \mid 0,0)=0$ implying that a system with one absorbing state indeed renormalizes into another one with the same property.

We have iterated equation (19) numerically using approximations of order $n=1,2,3,4,5$ and 8 . For all orders of approximation that we used we have found, in the plane $\left(p_{1}, p_{2}\right)$, two fully attractive fixed points $(0,0)$ and $(1,1)$, one fully repulsive fixed point $(1 / 2,1)$ and one nontrivial fixed point $\left(p_{1}^{*}, p_{2}^{*}\right)$. The attractive fixed point $(0,0)$ is related to the absorbing state whereas the other attractive fixed point $(1,1)$ is related to the active state. Almost all trajectories are attracted to either one or the other of these two points as can be seen in figure 1. The base of attraction are separated by a line, which should be identified as the critical line of the Domany-Kinzel model. The separatrix hits the line $p_{2}=1$ at $p_{1}=1 / 2$, which is a fully repulsive fixed point, and the line $p_{2}=0$ at $p_{1}=p_{c}$.

The nontrivial fixed point is located over the separatrix line and is attractive along a direction parallel to the separatrix and repulsive in the direction perpendicular (a hyperbolic fixed point). According to the $\mathrm{RG}$ theory the critical exponent $\nu_{\perp}$, related to the correlation length, is obtained from the eigenvalue $\lambda$ along the repulsive direction through $\nu_{\perp}=\ln b / \ln \lambda$. The eigenvalue $\lambda$ is the largest eigenvalue of the Hessian matrix related to the transformation $\left(p_{1}, p_{2}\right) \rightarrow\left(p_{1}^{\prime}, p_{2}^{\prime}\right)$ calculated at the nontrivial fixed point. The location of the critical line (the separatrix) is in fair agreement with previous results coming from numerical simulation [13], as can be seen in figure 2 . The critical value $p_{c}=0.792$ of $p_{1}$ at $p_{2}=0$ differs from the value $p_{c}=0.799 \pm 0.002$ obtained from numerical simulations [13] in about $1 \%$.

Table 1 shows the values of the critical point $p_{c}$ (along $p_{2}=0$ ) for several values of the order of approximation up to $n=8$. For $n \geq 4$ the figures are very close to the value obtained from simulations. In the same table it is shown the values of the nontrivial fixed point together with the eigenvalue $\lambda$ 
and the critical exponent $\nu_{\perp}=\ln 2 / \ln \lambda$. All figures for the eigenvalue $\lambda$ were obtained numerically since it is very difficult to calculated the derivatives of the Hessian matrix related to the transformation $\left(p_{1}, p_{2}\right) \rightarrow\left(p_{1}^{\prime}, p_{2}^{\prime}\right)$. The best value of the critical exponents, that were obtained from the approximation of order $n=8$, is $\nu_{\perp}=1.013$ which should be compared to $\nu_{\perp}=1.100 \pm 0.005$ obtained from transfer matrix methods [1].

\section{Conclusion}

We used a real space RG to obtain the critical behavior of the Domany-Kinzel cellular automaton. The phase diagram is the same as the one obtained from simulation. The critical line, with the exception of the point $(1 / 2,1)$, is dominated by just one nontrivial fixed point implying, within the ideas of $\mathrm{RG}$, that all critical points along the line are in the same universality class, as expected. The location of the critical line is in good agreement with previous results, the value of $p_{1}$ at $p_{2}=0$ differing in about $1 \%$ from the value obtained from numerical simulations. The best value of the critical exponents is in fair agreement with the values obtained from transfer matrix methods and from numerical simulations. A way of improving these figures is to use a renormalization scheme for which the renormalization factor $b$ is less than two. This could be accomplished, for instance, by renormalizing a block of three sites into a block of two in which case we would have $b=3 / 2$. Another way of getting better values is to use a $R G$ space with more parameters instead of just two as we have done here. 


\section{References}

[1] E. Domany and W. Kinzel, Phys. Rev. Lett. 53, 311 (1984)

[2] T. M. Liggett, Interacting Particle Systems, Springer-Verlag, New York, 1985.

[3] H. K. Janssen, Z. Phys. B 42, 151 (1981).

[4] P. Grassberger, Z. Phys. B 47, 365 (1982).

[5] Th. Niemeyer and J. M. J. van Leeuwen, Physica 71, 17 (1974).

[6] G. F. Mazenko, M. J. Nolan and O. T. Valls, Phys. Rev. Lett. 41, 500 (1978).

[7] G. F. Mazenko and O. T. Valls, Phys. Rev. B 31, 1565 (1985).

[8] F. Haake and M. Lewenstein, Phys. Rev. B 27, 5868 (1983).

[9] L. Pietronero, A. Vespignani and S. Zapperi, Phys. Rev. Lett. 72, 1690 (1994).

[10] A. Vespignani, S. Zapperi and L. Pietronero, Phys. Rev. E 51, 1711 (1995).

[11] A. Vespignani, S. Zapperi and V. Loreto, Phys. Rev. Lett. 77, 4560 (1996).

[12] F. Bagnoli, R. Bulajich, R. Livi and A. Maritan, J. Phys. A 25, L1071 (1992).

[13] G. F. Zebende and T. J. P. Penna, J. Stat. Phys. 74, 1273 (1994).

[14] R. Dickman and T. Tomé, Phys. Rev. A 44, 4833 (1991).

[15] D. ben-Avraham and J. Kohler, Phys. Rev. A 45, 8358 (1992). 


\section{Table caption}

Table 1. For each value $n$ of the order of approximation it is shown the critical point $p_{c}$ (along $p_{2}=0$ ), the nontrivial fixed point $\left(p_{1}^{*}, p_{2}^{*}\right)$, the eigenvalue $\lambda$ corresponding to this fixed point along the relevant direction and the critical exponent $\nu_{\perp}=\ln 2 / \ln \lambda$, related to the correlation length. 
Table 1

$\begin{array}{llllll}n & p_{c} & p_{1}^{*} & p_{2}^{*} & \lambda & \nu_{\perp} \\ 1 & 0.745782 & 0.595189 & 0.826419 & 2.118 & 0.924 \\ 2 & 0.778384 & 0.598983 & 0.927874 & 2.144 & 0.909 \\ 3 & 0.788641 & 0.591302 & 0.949335 & 2.034 & 0.976 \\ 4 & 0.792203 & 0.591732 & 0.946004 & 2.000 & 1.000 \\ 5 & 0.791504 & 0.591872 & 0.945275 & 1.988 & 1.009 \\ 8 & 0.791654 & 0.591930 & 0.944997 & 1.982 & 1.013\end{array}$




\section{Figure Caption}

Figure 1. Renormalization group trajectories in the parameter space $\left(p_{1}, p_{2}\right)$ of the Domany-Kinzel cellular automaton, obtained by using the approximation of order $n=4$. Almost all trajectories flow either to one of two fully atractive fixed points. One of them is related to the active state (open circle) and the other to the absorbing state (origin of coordinates). The two base of atraction are separated by a line, identified with the critical line of the model, where the hyperbolic nontrivial fixed point (full circle) and a fully repulsive point (star) are located.

Figure 2. Phase diagram of the Domany-Kinzel probabilistic cellular automaton showing the frozen absorbing state and the active state. The squares are results coming from numerical simulations [13] and the line is the result obtained from the present real space renormalization group using the approximation of order $n=4$. 
Figure 1. Tome and de Oliveira

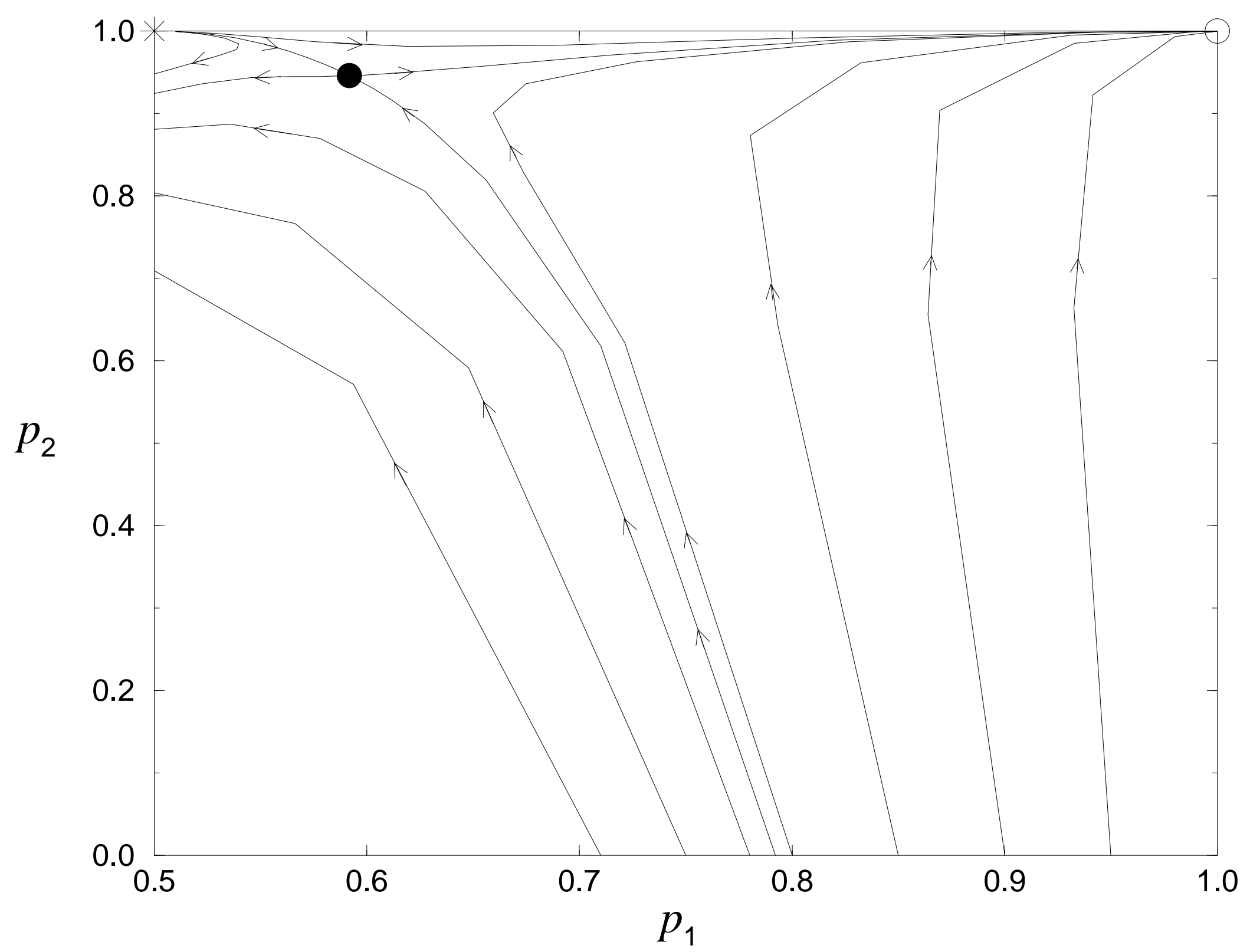


Figure 2. Tome and de Oliveira

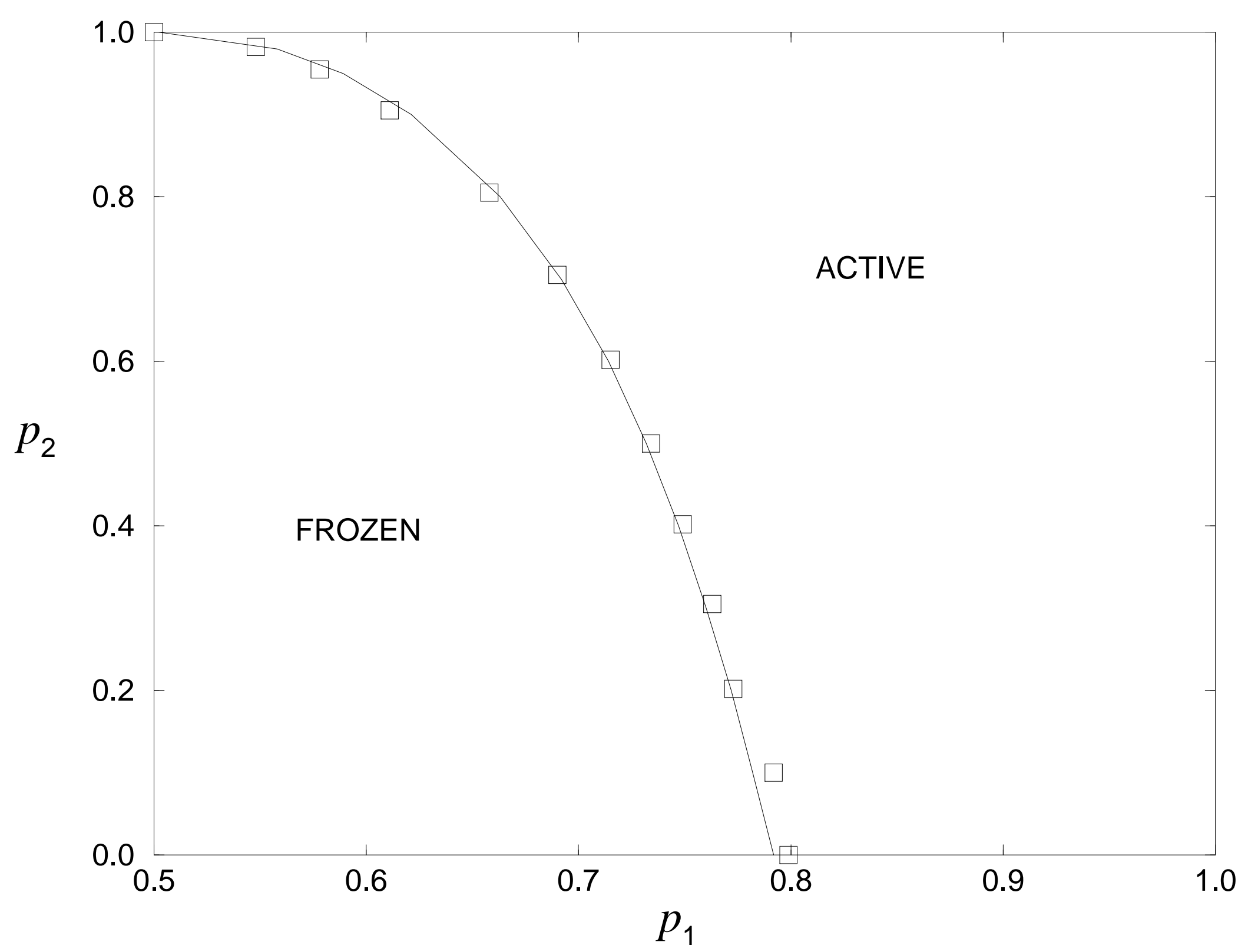

